Stochastic model for the epitaxial growth of two-dimensional islands in the submonolayer regime

This content has been downloaded from IOPscience. Please scroll down to see the full text.

J. Stat. Mech. (2016) 033211

(http://iopscience.iop.org/1742-5468/2016/3/033211)

View the table of contents for this issue, or go to the journal homepage for more

Download details:

This content was downloaded by: kargovsky

IP Address: 93.180.54.177

This content was downloaded on 22/03/2016 at 12:35

Please note that terms and conditions apply. 


\title{
Stochastic model for the epitaxial growth of two-dimensional islands in the submonolayer regime
}

\section{A V Kargovsky ${ }^{1}$, E I Anashkina ${ }^{1,2}$, O A Chichigina ${ }^{1}$, D Valenti ${ }^{2,3}$ and B Spagnolo ${ }^{2,3}$}

${ }^{1}$ Faculty of Physics and International Laser Center, Lomonosov Moscow State University, Leninskie Gory, 119992 Moscow, Russia

2 Dipartimento di Fisica e Chimica, Group of Interdisciplinary Theoretical Physics, Università di Palermo and CNISM, Unità di Palermo, Viale delle Scienze, Edificio 18, 90128 Palermo, Italy

${ }^{3}$ Istituto Nazionale di Fisica Nucleare, Sezione di Catania, Italy

E-mail: kargovsky@yumr.phys.msu.ru

Received 6 July 2015, revised 1 December 2015

Accepted for publication 31 January 2016

Published 22 March 2016

Online at stacks.iop.org/JSTAT/2016/033211

doi:10.1088/1742-5468/2016/03/033211

\begin{abstract}
The diffusion-based growth of islands composed of clusters of metal atoms on a substrate is considered in the aggregation regime. A stochastic approach is proposed to describe the dynamics of island growth based on a Langevin equation with multiplicative noise. The distribution of island sizes, obtained as a solution of the corresponding Fokker-Planck equation, is derived. The time-dependence of island growth on its fractal dimension is analysed. The effect of mobility of the small islands on the growth of large islands is considered. Numerical simulations are in a good agreement with theoretical results.
\end{abstract}

Keywords: dendritic growth (theory), diffusion-limited aggregation (theory), stochastic processes (theory), diffusion 


\section{Contents}

1. Introduction 2

2. The diffusion model of island growth 4

2.1. Langevin equation for island size . . . . . . . . . . . . . . . 4

2.2. Fokker-Planck equation for island size distribution. . . . . . . . . . 6

3. Small island capture $\quad 8$

4. Numerical results 9

5. Further generalization $\quad \mathbf{1 3}$

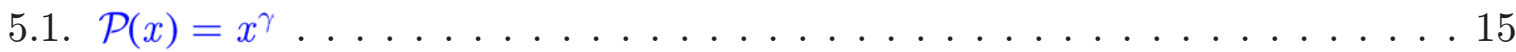

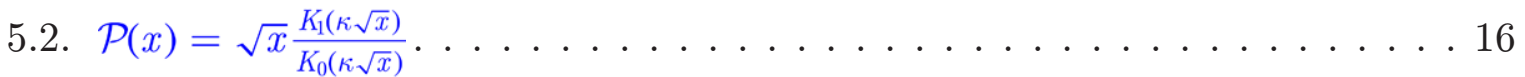

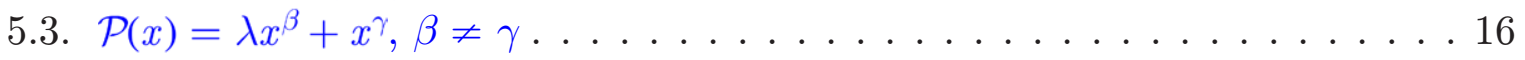

$\begin{array}{ll}\text { 6. Summary } & 18\end{array}$

$\begin{array}{lr}\text { Acknowledgments } & 18\end{array}$

$\begin{array}{ll}\text { References } & 18\end{array}$

\section{Introduction}

Modern technologies make possible the production of nanostructures on a substrate from preformed mass-selected clusters. Homogeneous nucleation and growth of islands from metal atoms during the initial submonolayer stage of film growth have been studied intensively for decades [1-5], although it is only recently that metal clusters became widely used in the formation of nanostructures [2, 6-9]. Specifically, clusters contain from several hundreds to several thousands of atoms and can be considered as classical particles. Within the context of nanotechnologies, production of nanostructures by diffusion is a subject largely investigated [10-12]. Moreover, during the last fifteen years, experimental techniques have allowed for the deposition of mass-selected clusters on surfaces [13-15]. Here, we are interested in cases when very low-energy neutral clusters produced in inert gas condensation sources [6] are mass-selected and deposited on a substrate.

Two-dimensional islands can be used for the fabrication of quantum dots [16]. Moreover, islands can form porous structures that are useful in the production of the chemical catalysts and sensors for the detection of chemical elements or viruses [17]. Each of these applications requires definite island shape, size, and density.

The density of islands and their sizes are determined by the relation between the diffusion coefficient and cluster flux. Clusters are deposited on a substrate and start to diffuse. They can stick together and form an island. The shape of the island depends on 
the chemical properties of cluster and substrate, and can vary from dendritic to compact, being connected with the fractal dimension of the island. At a relatively low flux or high diffusion coefficient a cluster may join an existing island before being trapped by another single cluster. Islands are nucleated predominantly at surface defects. Such a process is known as heterogeneous nucleation [18], and in this case large islands form on the surface. An increase in the flux or a decrease in diffusion can lead to an increase in the probability of several clusters sticking together and forming a new island rather than joining existing islands. Therefore, the number of islands grows, and the size of each island is small. Such a process is homogeneous $[18,19]$. In this work we are interested in structure formation on highly oriented pyrolytic graphite (HOPG) surfaces $[6,18,20,21]$, where diffusion is high and large branching islands grow. Since these structures are composed of metal clusters, we consider heteroepitaxial growth.

The two most common classes of method of description of epitaxial growth are (1) the analytic-based method, i.e. homogeneous rate equations (RE) $[1,22,23]$ and continuum models [24, 25], and (2) numerical atomistic models, such as molecular dynamics (MD) and kinetic Monte Carlo (KMC) techniques [26]. The standard RE approach is mostly based on mean field (MF) theory, but in general it does not lead to realistic island size distributions. This failure can be traced to the fact that the usual MF theory does not include correlations between the size of an island and its local environment [5, 27-31]. Such correlations are especially important in two dimensions, as in the island growth on surfaces. Several models addressed the description of non-MF behaviour and the propensity for an island to capture a diffusing cluster (i.e. capture number) based on modified RE [32] and RE for the joint probability distribution for island size $[28,33,34]$. The geometry-based simulation approach for submonolayer film growth [35] has to be mentioned, and also that the model of island dynamics for epitaxial growth is based on the level-set method [36-38]. Recently, a review of basic theoretical concepts to describe monolayer growth kinetics under non-equilibrium conditions was given in [9]. Features of the island distribution on a surface are considered in [39-41].

Note also that several attempts to obtain analytical expressions for the size-distribution of islands consisting of adatoms were pursued in [5, 42-44]. There are several examples of the application of the Fokker-Planck equation to describe the growth of nanostructures in the 3D case [8, 45, 46]. However, certain problems with the description of the nucleation process by the Fokker-Planck equation are noticed [47]. The coverage-dependent mean island size has a power-law dependence on coverage over much of the aggregation regime $[48,49]$. More exact models show that such a powerlaw dependence is only true approximately in the large coverage limit $[42,50]$, but that these time dependencies are qualitative. However, dynamic scaling of the island size-distribution in submonolayer epitaxial growth has been theoretically investigated in [4], and the coarsening effect on island-size scaling in adatoms was experimentally analysed in [51].

Cluster diffusion and aggregation of islands were studied using MD $[52,53]$, but this approach becomes time-consuming when applied to structures consisting of thousands of clusters. However, KMC simulations look more favourable [17, 54].

At the beginning of structure formation all islands grow by capturing clusters, and the number of islands also increases. At higher coverage the aggregation of islands starts, and the number of islands is stabilized. The probability of aggregation of two 
islands is defined by two processes. The first is the movement of the boundary of the growing large island that reaches unmoving small islands. The second is the arrival of small islands to the boundary of large ones. The influence of the mobility of small islands on the island size distribution can be neglected for adatoms [55] but not for large clusters on HOPG. High lubricity is typical for a HOPG surface, so it is necessary to analyse the diffusion of small islands while describing the island growth on this surface. Such mesoscopic objects as graphite flakes [56] and conglomerates of clusters [57] participate in thermal motion. They can demonstrate ballistic friction at high speed with complicated interplay of rotations and translations [58]. While describing the island growth, we neglect the internal structure of a small island and consider it as a structureless classical particle. As a result, in this paper we use the term 'particles' to characterize both captured islands and clusters.

Although much work has been done in this general area of investigation of epitaxial growth of two-dimensional islands in submonolayers, there is a lack of investigation into the effect of noise due to the diffusion capture. In fact, the diffusion-based capture of particles by islands is a stochastic process. In this paper, we analytically consider island size as a non-stationary random value. The mechanism for island growth is the Smoluchowski ripening. In particular, islands are allowed to diffuse, and they irreversibly grow upon collision [59-61]. We do not consider the nucleation regime. The surface density of islands reaches a maximum and remains almost constant for some ranges of coverage (e.g. a coverage around $15 \%$ is mentioned in [44]). We are interested in the aggregation regime, before coalescence and percolation, after the number density of islands has reached a saturation value, when islands grow both by the joining of small islands and clusters. The growth of large islands, due to the capturing of small islands by large ones, can be effectively described as a stochastic process. Specifically, the variation in the number of particles in a large island is described by a Langevin equation.

We analyse the influence of the noise properties on the probability distribution of island size using different kinds of noise source. This allows us to also consider the effect of rare but large fluctuations, which are important for the considered dynamics of island growth [9]. We obtain analytical expressions for the island size distribution and the mean island size from the corresponding Fokker-Planck equation. We find a good agreement between numerical simulations and theoretical results.

\section{The diffusion model of island growth}

\subsection{Langevin equation for island size}

To describe an island size we use the number of clusters in the island $N(t)$. We assume that, after joining a two-dimensional island, a particle is embedded in the island structure and cannot leave it. In addition, we disregard partial dissociation of the island. Moreover, the motion of the large island is not considered (see [20] for an experimental justification). We focus on the stage of the structure growth when islands are large and branching. The nucleation process is not considered. We assume that, if the particle is deposited on the island, it will diffuse to the boundary and fall over the edge to increase the size of the island. 
The island growth is caused by the total particle flux through its boundary $\dot{N}=\Pi(N, t)$. In $[1,43,44,62-64]$ authors considered a single, motionless circular island, which grows by absorption of diffusing particles on a $2 \mathrm{D}$ substrate. A quasistatic approximation is used for the analysis of the particle concentration since the change of island boundary is negligible at mean time of particle diffusion. The mean total flux of particles through the island boundary can be written as

$$
\bar{\Pi}=2 \pi R J \sqrt{D \tau} \frac{K_{1}\left(\frac{R}{\sqrt{D \tau}}\right)}{K_{0}\left(\frac{R}{\sqrt{D \tau}}\right)},
$$

where $D$ is the diffusion coefficient, $J$ is the incident particle flux, $R$ is the island radius (which is proportional to $\sqrt{N}$ ), $\tau$ is the mean time of particle motion, and $K_{\nu}(z)$ is the modified Bessel function. We can simplify equation (1) under the condition that cluster displacements are much shorter than the island radius $R \gg \sqrt{D \tau}$. This condition is settled in the case of high coverage with large branching islands. Then, we have

$$
\bar{\Pi}=P J \sqrt{D \tau},
$$

where $P$ is the island perimeter. In general, $\tau$ is time-dependent, but we assume that it depends weakly on time, and we consider $\tau$ as a constant value.

For non-circular islands, it is sufficient to replace the radius $R$ in equation (1) with an effective one, appropriate to the island morphology [43]. At low temperatures (or no edge diffusion), when the islands are ramified, the effective radius has a power-law dependence on the island area $s$ given by

$$
R_{\text {eff }} \sim s^{\gamma} \sim N^{\gamma}
$$

which is proportional to the island perimeter. Here, the exponent $\gamma \in[1 / 2,1)$ characterizes branching. If $\gamma \rightarrow 1 / 2$, the island is compact. If $\gamma \rightarrow 1$, the dendritic structure has thin branches, and the thickness of each branch approaches the width of a single cluster. Note that $\gamma^{-1}$ gives a measure of the fractal dimension.

Thus, the island growth depends on its boundary length since particles stick only to the boundary of an island and we write, finally

$$
\bar{\Pi} \sim N^{\gamma} J \sqrt{D \tau}
$$

In general, the growth rate dependence on the island size is more complicated than a simple proportionality to the perimeter. Also, we assume that, at the considered stage of growth, $\gamma$ is constant. For a dendritic structure, this means that the mean thickness of branches grows together with the island. Therefore, a stochastic differential equation for the island size is written as

$$
\dot{N}=N^{\gamma} \xi(t),
$$

where $\xi(t)$ is a noise source determined by the particle flux and the diffusion coefficient of a particle on the substrate. Since we consider irreversible growth, the variation of the island size can not be negative. Therefore, we use a non-negative noise source [65-67]. Below we interpret equation (5) in the Stratonovich sense [68, 69]. 


\subsection{Fokker-Planck equation for island size distribution}

Let us consider the following Langevin equation:

$$
\dot{x}=x^{\gamma} \xi(t), \quad x \in \mathbb{R}^{+},
$$

where $\xi(t)$ is the stationary Gaussian white noise with $\langle\xi(t)\rangle=a>0$ and $\langle\xi(t) \xi(t+\tau)\rangle=2 K \delta(\tau)$.

The corresponding Fokker-Planck equation for the probability density function (PDF) $w(x, t)$ can be written as [68]

$$
\frac{\partial w}{\partial t}=-\frac{\partial}{\partial x}\left(a x^{\gamma}+K \gamma x^{2 \gamma-1}\right) w+K \frac{\partial^{2}}{\partial x^{2}} x^{2 \gamma} w .
$$

The function $w(x, t)$ satisfies the initial and boundary conditions

$$
\begin{aligned}
w(x, 0) & =\psi(x), \\
{\left[\left(a x^{\gamma}-K \gamma x^{2 \gamma-1}\right) w-K x^{2 \gamma} \frac{\partial w}{\partial x}\right]_{x=0} } & =0, \\
\lim _{x \rightarrow \infty}\left[\left(a x^{\gamma}-K \gamma x^{2 \gamma-1}\right) w-K x^{2 \gamma} \frac{\partial w}{\partial x}\right] & =0 .
\end{aligned}
$$

Here, $\psi(x)$ is a non-negative function that satisfies the normalization condition and provides consistency of the initial and boundary conditions. In accordance with the boundary conditions, the probability current vanishes at the boundaries $x=0$ and $x=\infty$.

Using the change of variable $\eta=x^{1-\gamma} /(1-\gamma)$, we reduce equation (7) to

$$
\frac{\partial w}{\partial t}=-a \frac{\partial w}{\partial \eta}+K \frac{\partial^{2} w}{\partial \eta^{2}}
$$

The corresponding initial and boundary conditions are given by

$$
\begin{aligned}
w(\eta, 0)=\chi(\eta) & \equiv \psi(x(\eta))[(1-\gamma) \eta]^{\frac{\gamma}{1-\gamma}}, \\
{\left[\frac{\partial w}{\partial \eta}-\frac{a w}{K}\right]_{\eta=0} } & =0, \\
\lim _{\eta \rightarrow \infty}\left[\frac{\partial w}{\partial \eta}-\frac{a w}{K}\right] & =0 .
\end{aligned}
$$

A solution to the Robin boundary-value problem for parabolic equation (9) with conditions (10) can be obtained using the Laplace transform [70]. Changing the variable back to $x$, we obtain the solution to equation (7):

$$
\begin{aligned}
w(x, t)= & x^{-\gamma} \int_{0}^{\infty}\left[\frac{1}{\sqrt{4 \pi K t}} \mathrm{e}^{-\frac{a^{2} t}{4 K}+\frac{a\left(x^{1-\gamma}-\zeta^{1-\gamma}\right)}{2 K(1-\gamma)}}\left(\mathrm{e}^{-\frac{\left(x^{1-\gamma}-\zeta^{1-\gamma}\right)^{2}}{4 K(1-\gamma)^{2} t}}+\mathrm{e}^{-\frac{\left(x^{1-\gamma}+\zeta^{1-\gamma}\right)^{2}}{4 K(1-\gamma)^{2} t}}\right)\right. \\
& \left.-\frac{a}{2 K} \mathrm{e}^{\frac{a x^{1-\gamma}}{K(1-\gamma)}} \operatorname{erfc}\left(\frac{a(1-\gamma) t+x^{1-\gamma}+\zeta^{1-\gamma}}{\sqrt{4 K(1-\gamma)^{2} t}}\right)\right] \psi(\zeta) \mathrm{d} \zeta .
\end{aligned}
$$


Let us derive an analytical expression for the moments of $x$. Integration with respect to $x$ of the product of the power function and the first two terms of the integrand of the PDF (11) can be easily performed [71]. We integrate the third term of the integrand by parts using expansion of the incomplete gamma function [72]

$$
\begin{aligned}
M_{n}(t)= & \frac{1}{\sqrt{2 \pi}}\left[2 K(1-\gamma)^{2} t\right]^{\frac{n}{2-2 \gamma}} \Gamma\left(\frac{n}{1-\gamma}+1\right) \\
& \times \int_{0}^{\infty}\left[\mathrm{e}^{-\frac{\left(a(1-\gamma) t+\zeta^{1-\gamma}\right)^{2}}{8 K(1-\gamma)^{2} t}} D_{-\frac{n}{1-\gamma}}-1\left(-\frac{a(1-\gamma) t+\zeta^{1-\gamma}}{\sqrt{2 K(1-\gamma)^{2} t}}\right)\right. \\
& +\mathrm{e}^{-\frac{\left(a(1-\gamma) t-\zeta^{1-\gamma}\right)^{2}}{8 K(1-\gamma)^{2} t}-\frac{a \zeta^{1-\gamma}}{K(1-\gamma)}} \\
& \left.\times \sum_{k=0}^{\infty}\left(-\frac{2 a t}{\sqrt{2 K t}}\right)^{k} D_{-\frac{n}{1-\gamma}-1-k}\left(-\frac{a(1-\gamma) t-\zeta^{1-\gamma}}{\sqrt{2 K(1-\gamma)^{2} t}}\right)\right] \psi(\zeta) \mathrm{d} \zeta,
\end{aligned}
$$

where $D_{\nu}(z)$ is the parabolic cylinder function. We assume that the initial distribution is such that integrals (11) and (12) converge (e.g. $\psi(x) \in \mathrm{L}^{2}\left(\mathbb{R}^{+}\right)$).

If the initial condition is a delta function $w(x, 0)=\delta\left(x-x_{0}\right)$, the PDF (11) is transformed into

$$
\begin{aligned}
w(x, t)= & \frac{x^{-\gamma}}{\sqrt{\pi K t}} \mathrm{e}^{-\frac{\left(a(1-\gamma) t-x^{1-\gamma}+x_{0}^{1-\gamma}\right)^{2}+2\left(x x_{0}\right)^{1-\gamma}}{4 K(1-\gamma)^{2} t} \cosh \left(\frac{\left(x x_{0}\right)^{1-\gamma}}{2 K(1-\gamma)^{2} t}\right)} \\
& -\frac{a x^{-\gamma}}{2 K} \mathrm{e}^{\frac{a x^{1-\gamma}}{K(1-\gamma)}} \operatorname{erfc}\left(\frac{a(1-\gamma) t+x^{1-\gamma}+x_{0}^{1-\gamma}}{\sqrt{4 K(1-\gamma)^{2} t}}\right)
\end{aligned}
$$

and coincides with the distribution obtained in [73] for $a=0$. Then, the expression of the mean of $x(t)$ is

$$
\begin{aligned}
\bar{x}(t)= & \frac{1}{\sqrt{2 \pi}}\left[2 K(1-\gamma)^{2} t\right]^{\frac{1}{2-2 \gamma}} \Gamma\left(\frac{2-\gamma}{1-\gamma}\right) \\
& \times\left[\mathrm{e}^{-\frac{\left(a(1-\gamma) t+x_{0}^{1-\gamma}\right)^{2}}{8 K(1-\gamma)^{2} t}} D_{\frac{\gamma-2}{1-\gamma}}\left(-\frac{a(1-\gamma) t+x_{0}^{1-\gamma}}{\sqrt{2 K(1-\gamma)^{2} t}}\right)\right. \\
& \left.+\mathrm{e}^{-\frac{\left(a(1-\gamma) t-x_{0}^{1-\gamma}\right)^{2}}{8 K(1-\gamma)^{2} t}-\frac{a x_{0}^{1-\gamma}}{K(1-\gamma)}} \sum_{k=0}^{\infty}\left(-\frac{2 a t}{\sqrt{2 K t}}\right)^{k} D_{\frac{\gamma-2}{1-\gamma}-k}\left(-\frac{a(1-\gamma) t-x_{0}^{1-\gamma}}{\sqrt{2 K(1-\gamma)^{2} t}}\right)\right] .
\end{aligned}
$$

For large time $t \gg \frac{x_{0}^{1-\gamma}}{a(1-\gamma)}$, the information about the initial distribution is lost, and the PDF tends to

$$
w(x, t)=\frac{x^{-\gamma}}{\sqrt{4 \pi K t}} \mathrm{e}^{-\frac{\left(x^{1-\gamma}-a(1-\gamma) t\right)^{2}}{4 K(1-\gamma)^{2} t}}\left[1-\frac{1}{2} \operatorname{erfc}\left(\frac{a t}{\sqrt{4 K t}}\right)\right]^{-1} .
$$

Then, the asymptotic expressions of the mean and variance are respectively 


$$
\bar{x}(t)=[a(1-\gamma) t]^{\frac{1}{1-\gamma}}
$$

and

$$
\sigma^{2}(t)=\frac{2 K}{a(1-\gamma)}[a(1-\gamma) t]^{\frac{1+\gamma}{1-\gamma}} .
$$

If the initial distribution is localized at relatively small $x$, expression (11) is transformed into formula (15) for sufficiently large times.

If the island size $N$ is sufficiently large, the relative variation of $N^{\gamma}$ per unit step is small. As a consequence, the discrete process, the dynamics of which are described by the stochastic differential equation (5), can be approximated by the continuous one of equation (6).

\section{Small island capture}

Let a small island consisting of several clusters join the large island at time $t_{i}$. This process can be modelled as a pulse noise

$$
\vartheta(t)=\sum_{i} f_{i} \delta\left(t_{i}-t\right)
$$

Each pulse corresponds to the capturing of a particle (a cluster or a small island), the amplitude of the $i$-th pulse $f i$ is proportional to the number of clusters in a particle; therefore, $\dot{N}=\vartheta(t)$. This pulse noise is a Poisson process, and the distance between pulses is determined by the probability per unit of time of a particle appearance. This process is not stationary since the probability depends on the perimeter of the large island and, consequently, on island size in accordance with equation (4), and increases as $\nu N^{\gamma}$, where $\nu$ is constant. Since pulse process is delta-correlated, it is defined by two parameters: the mean value $\langle\vartheta\rangle=\langle f\rangle \nu N^{\gamma}$ and the constant spectral density $S_{\vartheta}=\left\langle f^{2}\right\rangle \nu N^{\gamma}$. Both these parameters are proportional to $N^{\gamma}$. Hence, we can replace additive non-stationary noise with multiplicative stationary noise $\vartheta(t)=N^{\gamma} \xi(t)$ with parameters $\langle\xi\rangle=\langle f\rangle \nu$ and $S_{\xi}=\left\langle f^{2}\right\rangle \nu$, and obtain equation (5). Mean time between pulses is denoted by $T$.

We take into account diffusion of small islands containing $\mathfrak{n}$ clusters. Let the size of the island, which is able to diffuse, be limited by the number $\mathcal{N}$. To analyse the effect of the mobility of small islands on the growth of the large island we consider that islands diffuse as clusters. The effects of cluster diffusion on the island size distribution have been reviewed for the case of irreversible growth of compact islands on a 2D substrate, and several mechanisms for the diffusion of large particles on solid surfaces were proposed [74-79]. In most cases, scaling arguments predict that the coefficient of particle diffusion decays as a power law with particle sizes $D \sim \mathfrak{n}^{-\mu}$. In particular, three different limiting cases were considered: (1) diffusion due to uncorrelated evaporation condensation $(\mu=1 / 2)$, which corresponds to the Brownian diffusion in two dimensions, (2) diffusion due to correlated evaporation condensation $(\mu=1)$, and (3) diffusion due to periphery diffusion $(\mu=3 / 2$ ). In a molecular dynamics study, Deltour et al found $\mu=2 / 3$ [80]. The model of Brownian diffusion is also suitable for the description of the 
motion of islands composed of clusters. In our model we consider two main processes for the growth of islands. The first one is the motion of the boundary of large islands. The probability of this kind of capturing of a particle is proportional to a certain parameter $c$ (see below). The second one is the diffusion of small islands arriving to the boundary of large ones. In accordance with the scaling power law mentioned above and equation (4), the probability of this kind of capturing of a particle is proportional to $\mathfrak{n}^{-\mu / 2}$. Summarizing, we have stationary pulse noise

$$
\xi(t)=f_{0} \sum_{i} \mathfrak{n}_{i} \delta\left(t_{i}-t\right)
$$

where the probability distribution of pulse amplitudes $\mathfrak{n}_{i}$ is

$$
P(\mathfrak{n})=\frac{1}{\mathcal{N} c+H_{\mathcal{N}}^{(\mu / 2)}}\left(c+\mathfrak{n}^{-\mu / 2}\right) .
$$

Here, $\left(\mathcal{N} c+H_{\mathcal{N}}^{(\mu / 2)}\right)^{-1}$ is a normalization factor, $H_{\mathcal{N}}^{(\nu)}=\sum_{k=1}^{\mathcal{N}} k^{-\nu}$ is the generalized harmonic number [81], and $\mathfrak{n} \leqslant \mathcal{N}$. A sample of pulse random process $\xi(t)$ of equation (19) is shown in figure 1.

At the same time, the probability of meeting a small island close to the boundary of large branching islands decreases with the increase in the size of the small island not only due to a decrease in mobility, but also because of competition. Moreover, in the work [82] another mechanism is discussed: 'The comparison between several kinetic models indicates that the correlation between the locations of the islands affects the coalescence rate. The fact that the islands "avoid" each other during the nucleation process lowers the rate of coalescence when the coverage is low. The onset of coalescence is pushed to higher coverage, and when the process finally begins, it will occur at a higher rate than in the case when the correlations are absent'. In this work, to model these correlations, which are actually anti-correlations, we use also the geometric probability distribution for the size of captured particles

$$
P(\mathfrak{n})=p(1-p)^{\mathfrak{n}-1} .
$$

This distribution corresponds to a faster decrease in the probability with size.

\section{Numerical results}

Here, we present results obtained by numerical integration of equation (5), using the pulse noise (19) with the two different amplitude distributions given in equations (20) and (21). The Mersenne twister [83] is used as a pseudo-random number generator. The averaging is performed over $10^{6}$ stochastic realizations in each analysed case. As the initial distribution, we use the generalized gamma distribution (see [4]) with critical island size $i$. In all subsequent calculations the mean pulse distance is $T=1$ and the pulse scale factor $f_{0}=0.001$. The pulse noise with a probability of 20 is characterized by the maximum number $\mathcal{N}$ of clusters in the captured island and exponent $\mu$. The value of $c$ is chosen in such a way that the probability is equal for two events: (a) the 


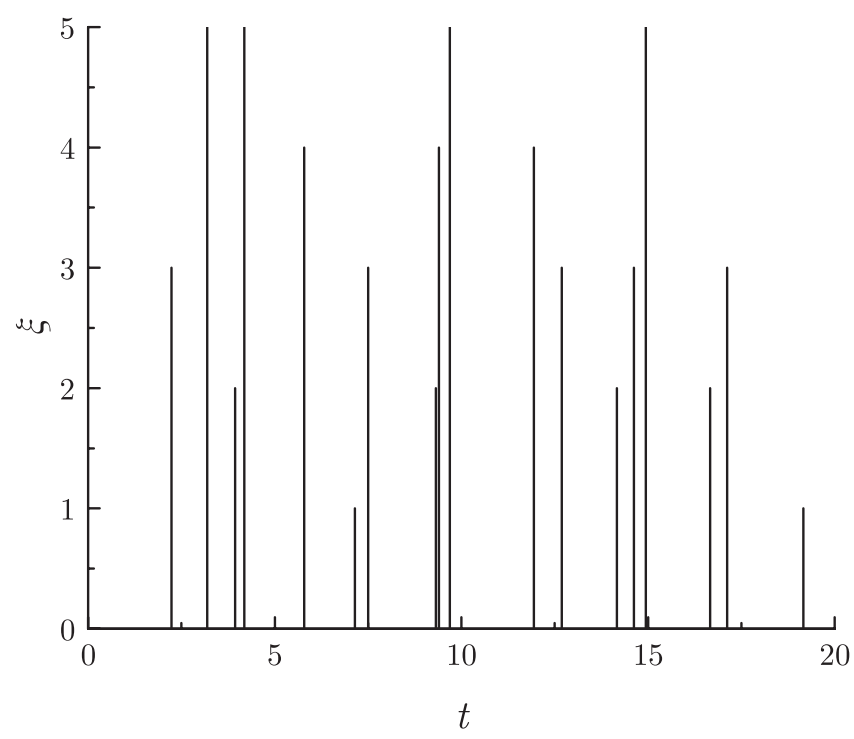

Figure 1. Sample of pulse random process $\xi(t)$ with amplitude pmf (20) ( $T=1$, $\left.f_{0}=1, \mathcal{N}=5, \mu=2 / 3, c=3^{-\frac{1}{3}}\right)$.

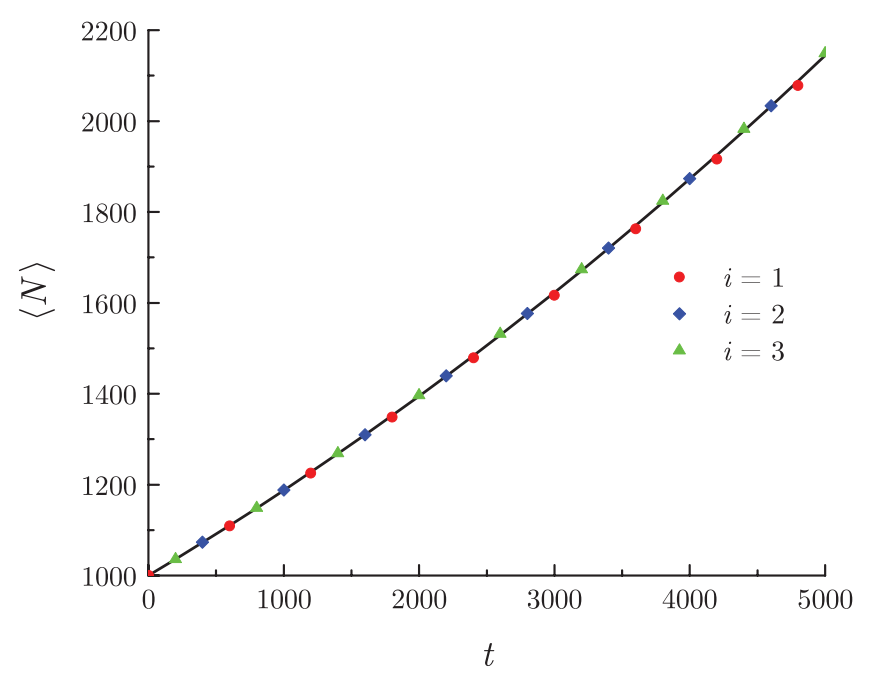

Figure 2. Plot of the mean island size versus time for different values of initial critical island size $i\left(\gamma=0.6, N_{0}=1000, \mathcal{N}=5, \mu=2 / 3\right)$ : results of analytical calculations (solid line) and numerical calculations (symbols) from equation (12) and equation (5), respectively.

capturing of a small island, consisting of three clusters, due to the moving boundary of a large island; (b) the capturing of this small island due to its movement towards the boundary of the large one.

The mean value in figure 2 and the variance in figure 3 were obtained for initial distributions (see [4]) with mean initial size $N_{0}=1000, \gamma=0.6$, noise parameters $\mathcal{N}=5$, and $\mu=2 / 3$, and different critical sizes $i=1,2,3$. We note that the overlap of the three curves of figure 2 and the non-overlap of the three curves of figure 3 can be explained noting that, as $i$ varies, the mean value of the initial distribution keeps constant, while the corresponding standard deviation changes. In other words, the value of $i$ does not influence the dynamics of the mean size, but affects the variance. 


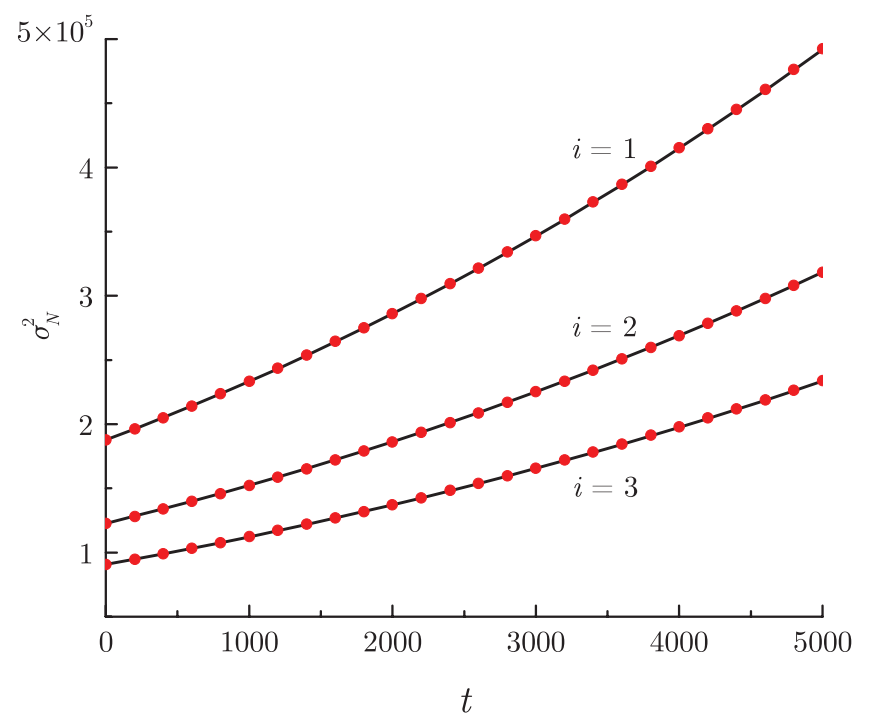

Figure 3. Plot of the variance of island size versus time for different values of initial critical island size $i\left(\gamma=0.6, N_{0}=1000, \mathcal{N}=5, \mu=2 / 3\right)$ : results of analytical calculations (solid lines) and numerical calculations (dots) (from equation (12) and equation (5), respectively).

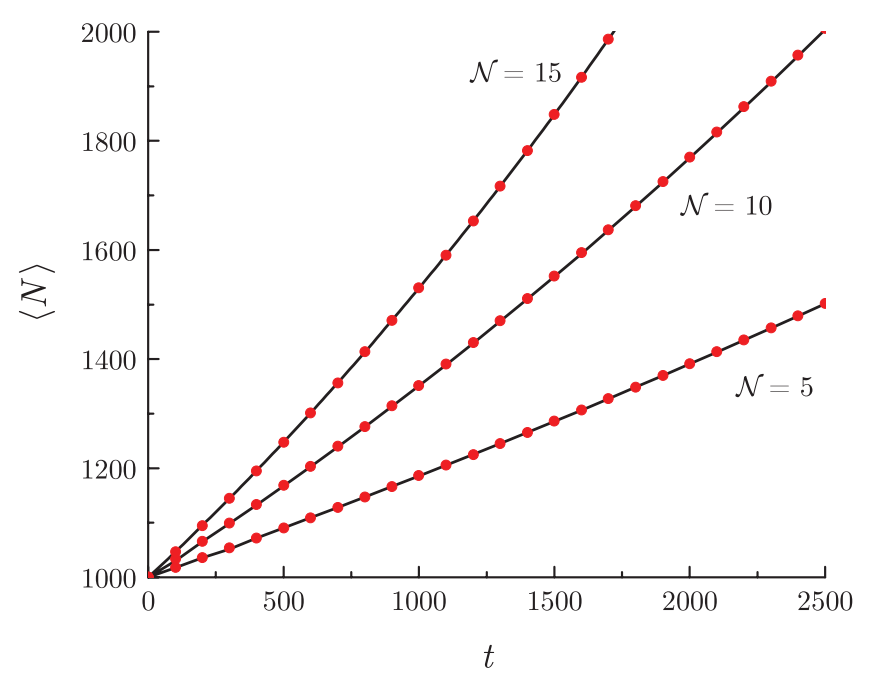

Figure 4. Plot of the mean island size versus time for different values of $\mathcal{N}(\gamma=0.6$, $\mu=2 / 3$, and initial distribution [4] with parameters $N_{0}=1000$ and $i=1$ ): results of analytical calculations (solid lines) and numerical calculations (dots) from equation (12) and equation (5), respectively.

We analyse the influence of change of noise parameters on the mean island size. In particular, figure 4 shows the effect of the number $\mathcal{N}$ on the island growth. We carried out the calculation for $\mathcal{N}=5,10,15$. The values of the other parameters are $\gamma=0.6, \mu=2 / 3$. Moreover, the initial distribution [4] with $N_{0}=1000$ and $i=1$. We see in figure 4 how the rate of growth of the mean value increases with $\mathcal{N}$. The result can be easily explained since $\mathcal{N}$ corresponds to the maximum size of a small island that joins the big island, and the increase of $\mathcal{N}$ allows the big island to capture larger particles. 


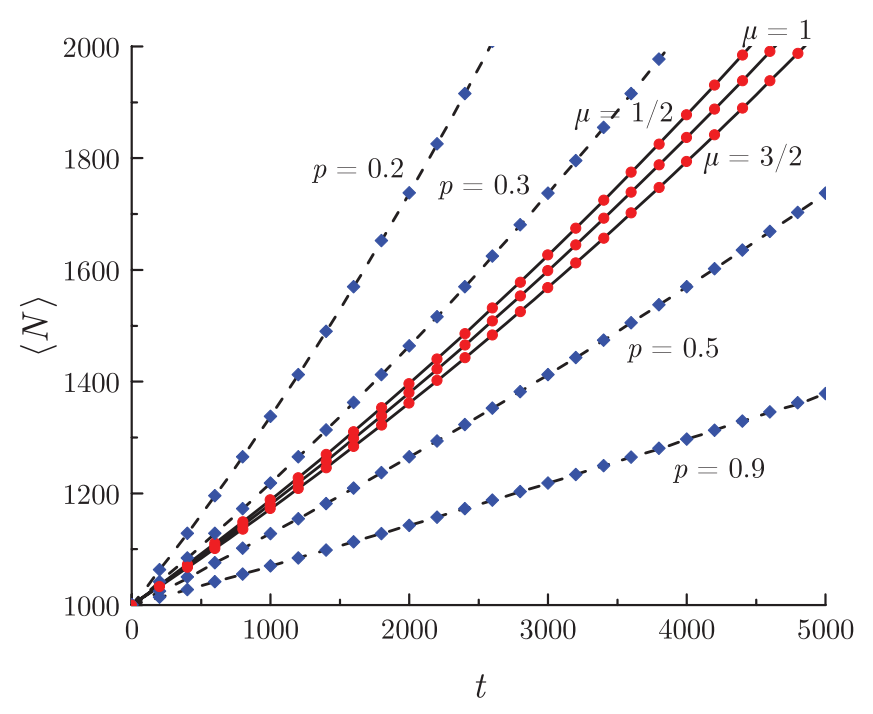

Figure 5. Plot of the mean island size versus time for different distributions of noise pulse amplitude $\left(\gamma=0.6\right.$, and initial distribution [4] with $N_{0}=1000$ and $\left.i=1\right)$ : results of analytical calculations (lines) and numerical calculations (symbols) from equation (12) and equation (5), respectively. There are probability distribution equation (20) with $N=5$ and $\mu=1 / 2,1,3 / 2$ (solid lines/dots); geometric probability distribution equation (21) with values of the parameter $p=0.2,0.3$, $0.5,0.9$ (dash lines/diamonds)

In figure 5 we present the time dynamics of the mean island size for the noise with distribution of amplitude presented in equation (20) (the value of exponent $\mu$, namely, $\mu=0.5, \mu=1$, and $\mu=1.5)$. The values of the other parameters are $\gamma=0.6, \mathcal{N}=5$, and initial distribution [4] with $N_{0}=1000$ and $i=1$. Here, the mean value of island size grows faster with decreasing $\mu$ and, consequently, with the increase of the probability of larger islands joining. This result can be also predicted noting that, since the diffusion coefficient increases with the decrease of $\mu$, the mean value of noise and, as a consequence, the growth rate are expected to increase. In the same figure we show results for pulse noise with geometric distribution of amplitude presented in equation (21), with parameters $p=0.2, p=0.3, p=0.5$ and $p=0.9$. Note that the increase of $p$ leads to the decrease in the growth rate of the island. This can be seen both from the numerical simulation and from the fact that with the increase of $p$, the mean value of noise, that is $1 / p$, decreases, thus, leading to the slowing of island growth.

Figure 6 illustrates the dependence of island growth on the degree of branching $\gamma$ of the island. We note that the island growth rate is much faster for large values of $\gamma$. Other parameters are $\mathcal{N}=5, \mu=2 / 3$, and initial distribution [4] with $N_{0}=1000$ and $i=1$. We can see that with the increase of branching of the island the rate of island growth also increases.

Figure 7 shows the time evolution of the island size distribution obtained from equation (11). Parameters of the simulation are $\gamma=0.6, \mathcal{N}=5, \mu=2 / 3$, and initial distribution [4] with $N_{0}=1000$ and $i=1$. The expected result was obtained: if we follow the time evolution of distribution of island size, we can see that the maximum of distribution shifts, and the distribution broadens.

We note that the analytical and numerical results are in a very good agreement. 


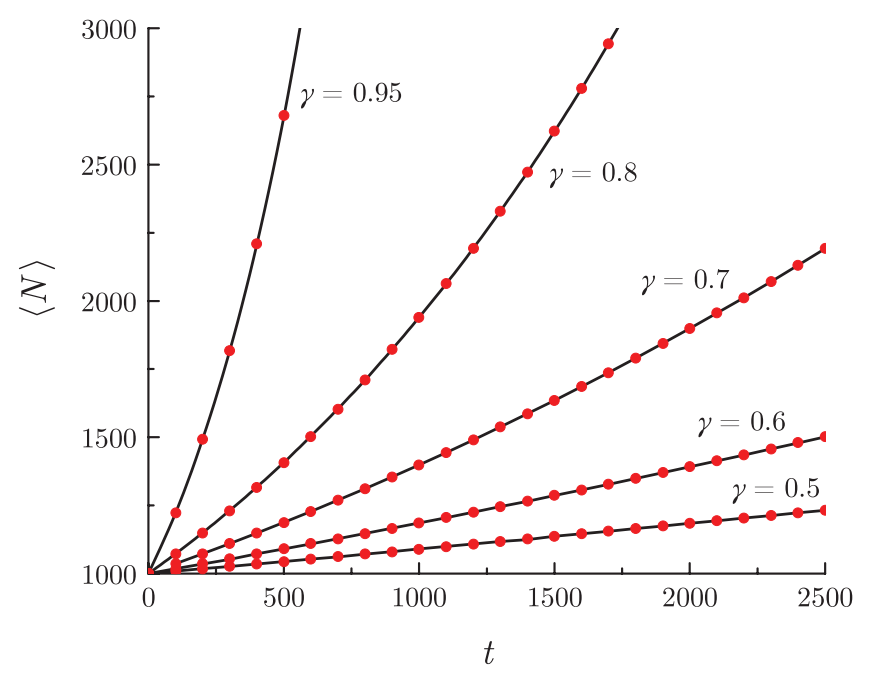

Figure 6. Plot of the mean island size versus time for different values of $\gamma(\mathcal{N}=5$, $\mu=2 / 3$, and initial distribution [4] with $N_{0}=1000$ and $i=1$ ): results of analytical calculations (solid lines) and numerical calculations (dots) from equation (12) and equation (5), respectively.

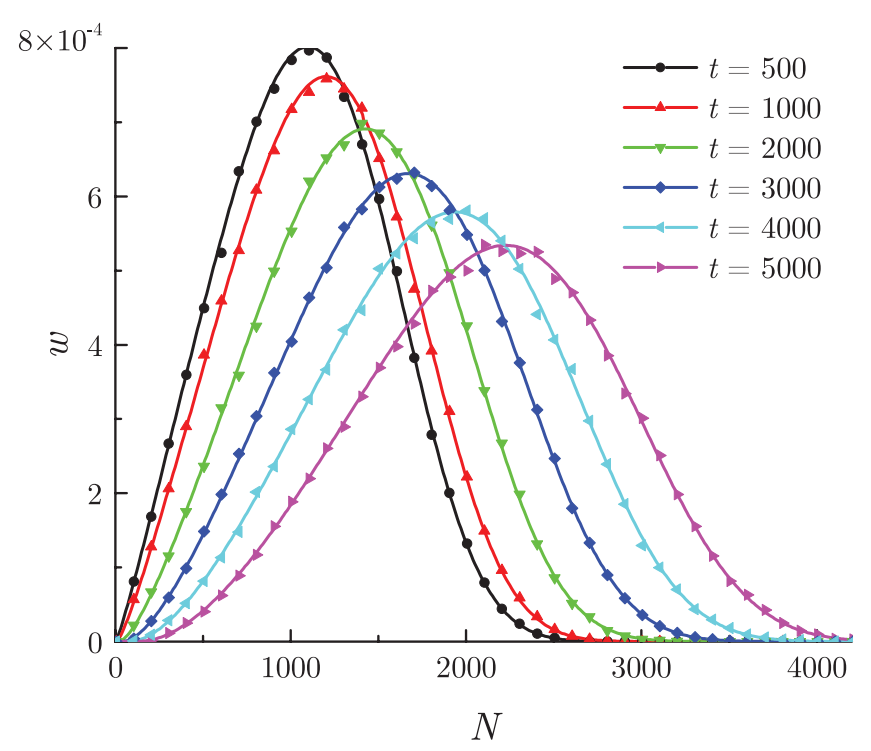

Figure 7. Time evolution of the island size distribution $(\gamma=0.6, \mathcal{N}=5$, $\mu=2 / 3$, and initial distribution [4] with $N_{0}=1000$ and $i=1$ ): results of analytical calculations (solid lines) and numerical calculations (symbols) from equation (11) and equation (5), respectively.

\section{Further generalization}

In this section, we can take into account several time-dependent factors (considered as constant parameters in previous sections) influencing island growth. In particular, these time-dependent factors are: the flux or temperature, the number of surrounding islands, and the growing nanostructures deforming the substrate. Here, we consider a more general Langevin equation: 


$$
\dot{x}=\frac{\mathcal{P}(x)}{\alpha(t)} \xi(t), \quad x \in \mathbb{R}^{+},
$$

where $\xi(t)$ is the stationary Gaussian white noise with $\langle\xi(t)\rangle=a>0$ and $\langle\xi(t) \xi(t+\tau)\rangle=2 K \delta(\tau), \mathcal{P}(x)$ is a general function of the geometric factors of the system investigated, and $\alpha(t)$ is a parameter which takes into account all of the time depending factors mentioned above.

For variable $\eta=\int \mathcal{P}^{-1}(x) \mathrm{d} x$, we get the Fokker-Planck equation

$$
\frac{\partial w}{\partial t}=-\frac{a}{\alpha(t)} \frac{\partial w}{\partial \eta}+\frac{K}{\alpha^{2}(t)} \frac{\partial^{2} w}{\partial \eta^{2}}
$$

The corresponding initial and boundary conditions are given by

$$
\begin{aligned}
w(\eta, 0)=\chi(\eta) & \equiv \psi(x(\eta))[(1-\gamma) \eta]^{\frac{\gamma}{1-\gamma}} \\
{\left[\frac{\partial w}{\partial \eta}-\frac{a \alpha(t) w}{K}\right]_{\eta=0} } & =0, \\
\lim _{\eta \rightarrow \infty}\left[\frac{\partial w}{\partial \eta}-\frac{a \alpha(t) w}{K}\right] & =0 .
\end{aligned}
$$

The solution to the Robin boundary-value problem with time-dependent coefficient for parabolic equation (23) and conditions (24) can be obtained using the single-layer potential [84]. The fundamental solution of equation (23) can be written as

$$
\begin{aligned}
\mathcal{E}(\eta, \lambda, t, \tau) & =\frac{1}{\sqrt{4 \pi K \theta_{2}(t, \tau)}} \mathrm{e}^{-\frac{\left(\eta-a \theta_{1}(t, \tau)-\lambda\right)^{2}}{4 K \theta_{2}(t, \tau)}}, t>\tau, \\
\theta_{n}(t, \tau) & =\int_{\tau}^{t} \frac{\mathrm{d} \tilde{t}}{\alpha^{n}(\tilde{t})} .
\end{aligned}
$$

Then, we have

$$
w(\eta, t)=\int_{0}^{\infty} \mathcal{E}(\eta, \lambda, t, 0) \chi(\lambda) \mathrm{d} \lambda+\int_{0}^{t} \mathcal{E}(\eta, 0, t, \tau) \phi(\tau) \mathrm{d} \tau,
$$

where the second term is the single-layer potential with density $\phi(t)$. The unknown function $\phi(t)$ is the solution of the Volterra integral equation of the second kind

$$
\phi(t)=\int_{0}^{t} \mathfrak{K}(t, \tau) \phi(\tau) \mathrm{d} \tau+f(t),
$$

where the kernel $\mathfrak{K}(t, \tau)$ and inhomogeneity $f(t)$ are given by

$$
\begin{aligned}
& \mathfrak{K}(t, \tau)=\frac{1}{\sqrt{\pi K \theta_{2}(t, \tau)}} \mathrm{e}^{-\frac{a^{2} \theta_{1}^{2}(t, \tau)}{4 K \theta_{2}(t, \tau)}\left(\frac{a \theta_{1}(t, \tau)}{2 K \theta_{2}(t, \tau)}-\frac{a \alpha(t)}{K}\right),} \\
& f(t)=\int_{0}^{\infty} \frac{1}{\sqrt{\pi K \theta_{2}(t)}} \mathrm{e}^{-\frac{\left(a \theta_{1}(t)+\lambda\right)^{2}}{4 K \theta_{2}(t)}}\left(\frac{a \theta_{1}(t)+\lambda}{2 K \theta_{2}(t)}-\frac{a \alpha(t)}{K}\right) \chi(\lambda) \mathrm{d} \lambda .
\end{aligned}
$$


Here, we use the notation $\theta_{n}(t) \equiv \theta_{n}(t, 0)$. The kernel (28) is weakly singular and, therefore, the solution of equation (27) may be obtained by a successive approximation (see [70]). The uniqueness of the solution is discussed in [84].

The solution of equation (23) is too cumbersome. However, we can determine an approximate solution for relatively large $t$. We assume that

$$
\lim _{t \rightarrow \infty} \frac{\theta_{1}^{2}(t)}{\theta_{2}(t)}=\infty .
$$

Then, the component of the probability current that corresponds to the first term of equation (26) tends to zero owing to the exponential function. Keeping in mind the normalization condition, we get expressions for the PDF

$$
\begin{aligned}
w(x, t)= & \frac{1}{\sqrt{4 \pi K \theta_{2}(t)} \mathcal{P}(x)} \int_{0}^{\infty} \exp \left(-\frac{\left[\int_{\zeta}^{x} \mathcal{P}^{-1}(\tilde{x}) \mathrm{d} \tilde{x}-a \theta_{1}(t)\right]^{2}}{4 K \theta_{2}(t)}\right) \\
& \times\left[1-\frac{1}{2} \operatorname{erfc}\left(\frac{a \theta_{1}(t)+\int_{0}^{\zeta} \mathcal{P}^{-1}(\tilde{x}) \mathrm{d} \tilde{x}}{\sqrt{4 K \theta_{2}(t)}}\right)\right]^{-1} \psi(\zeta) \mathrm{d} \zeta
\end{aligned}
$$

For significantly large times, which depend on the function $\alpha(t)$, the information on the initial distribution is lost, and the PDF tends to

$$
\begin{aligned}
w(x, t)= & \frac{1}{\sqrt{4 \pi K \theta_{2}(t)} \mathcal{P}(x)} \exp \left(-\frac{\left[\int_{0}^{x} \mathcal{P}^{-1}(\tilde{x}) \mathrm{d} \tilde{x}-a \theta_{1}(t)\right]^{2}}{4 K \theta_{2}(t)}\right) \\
& \times\left[1-\frac{1}{2} \operatorname{erfc}\left(\frac{a \theta_{1}(t)}{\sqrt{4 K \theta_{2}(t)}}\right)\right]^{-1} .
\end{aligned}
$$

Below we consider several kinds of function $\mathcal{P}(x)$, which may be useful in the analysis of island growth.

\section{1. $\mathcal{P}(x)=x^{\gamma}$}

This case corresponds to the system investigated in previous sections, when the growth rate is proportional to the island perimeter. As mentioned above, in this case we have $\eta=x^{1-\gamma} /(1-\gamma)$. Then, for the PDF we get

$$
\begin{aligned}
w(x, t)= & \frac{x^{-\gamma}}{\sqrt{4 \pi K \theta_{2}(t)}} \int_{0}^{\infty} \mathrm{e}^{-\frac{\left(x^{1-\gamma}-a(1-\gamma) \theta_{1}(t)-\zeta^{1-\gamma}\right)^{2}}{4 K(1-\gamma)^{2} \theta_{2}(t)}} \\
& \times\left[1-\frac{1}{2} \operatorname{erfc}\left(\frac{a(1-\gamma) \theta_{1}(t)+\zeta^{1-\gamma}}{\sqrt{4 K(1-\gamma)^{2} \theta_{2}(t)}}\right)\right]^{-1} \psi(\zeta) \mathrm{d} \zeta,
\end{aligned}
$$


and the moments

$$
\begin{aligned}
M_{n}(t)= & \frac{1}{\sqrt{2 \pi}}\left[2 K(1-\gamma)^{2} \theta_{2}(t)\right]^{\frac{n}{2-2 \gamma}} \Gamma\left(\frac{n}{1-\gamma}+1\right) \int_{0}^{\infty} \mathrm{e}^{-\frac{\left(a(1-\gamma) \theta_{1}(t)+\zeta^{1-\gamma}\right)^{2}}{8 K(1-\gamma)^{2} \theta_{2}(t)}} \\
& \times D_{-\frac{n}{1-\gamma}-1}\left(-\frac{a(1-\gamma) \theta_{1}(t)+\zeta^{1-\gamma}}{\sqrt{2 K(1-\gamma)^{2} \theta_{2}(t)}}\right)\left[1-\frac{1}{2} \operatorname{erfc}\left(\frac{a(1-\gamma) \theta_{1}(t)+\zeta^{1-\gamma}}{\sqrt{4 K(1-\gamma)^{2} \theta_{2}(t)}}\right)\right]^{-1} \psi(\zeta) \mathrm{d} \zeta .
\end{aligned}
$$

For significantly large times the PDF tends to

$$
w(x, t)=\frac{x^{-\gamma}}{\sqrt{4 \pi K \theta_{2}(t)}} \mathrm{e}^{-\frac{\left(x^{1-\gamma}-a(1-\gamma) \theta_{1}(t)\right)^{2}}{4 K(1-\gamma)^{2} \theta_{2}(t)}}\left[1-\frac{1}{2} \operatorname{erfc}\left(\frac{a \theta_{1}(t)}{\sqrt{4 K \theta_{2}(t)}}\right)\right]^{-1} .
$$

Similarly to equations (16) and (17), we derive the asymptotic expressions for mean and variance

$$
\begin{aligned}
\bar{x}(t) & =\left[a(1-\gamma) \theta_{1}(t)\right]^{\frac{1}{1-\gamma}}, \\
\sigma^{2}(t) & =2 K \theta_{2}(t)\left[a(1-\gamma) \theta_{1}(t)\right]^{\frac{2 \gamma}{1-\gamma}} .
\end{aligned}
$$

5.2. $\mathcal{P}(x)=\sqrt{x} \frac{K_{1}(\kappa \sqrt{x})}{K_{0}(\kappa \sqrt{x})}$

This kind of function $\mathcal{P}(x)$ arises from the mean field approximation of quasistatic growth of a circular island, when the cluster flux through the island boundary is

$$
\Pi(R)=2 \pi R J \sqrt{D \tau} \frac{K_{1}\left(\frac{R}{\sqrt{D \tau}}\right)}{K_{0}\left(\frac{R}{\sqrt{D \tau}}\right)} .
$$

Then, the steady-state PDF is

$$
\begin{aligned}
w(x, t)= & \frac{K_{0}(\kappa \sqrt{x})}{\sqrt{4 \pi x K \theta_{2}(t)} K_{1}(\kappa \sqrt{x})} \exp \left(-\frac{\left[-2 / \kappa \log \left[\kappa \sqrt{x} K_{1}(\kappa \sqrt{x})\right]-a \theta_{1}(t)\right]^{2}}{4 K \theta_{2}(t)}\right) \\
& \times\left[1-\frac{1}{2} \operatorname{erfc}\left(\frac{a \theta_{1}(t)}{\sqrt{4 K \theta_{2}(t)}}\right)\right]^{-1} .
\end{aligned}
$$

Figure 8 shows scaled steady-state PDF for different values of $\kappa$. The values of the parameters are $a=10^{-3}, K=10^{-4}$, and $t=10^{5}$. Here, we use the function $\alpha(t)=1$ and $\theta_{n}(t)=t$

\section{3. $\mathcal{P}(x)=\lambda x^{\beta}+x^{\gamma}, \beta \neq \gamma$}

The case $\beta=1$ corresponds to a beyond-mean-field approximation, when island growth is proportional to island area. When $\beta=0$, equation (22) describes island formation around a defect of the surface, and the size of the defect is not negligible. 


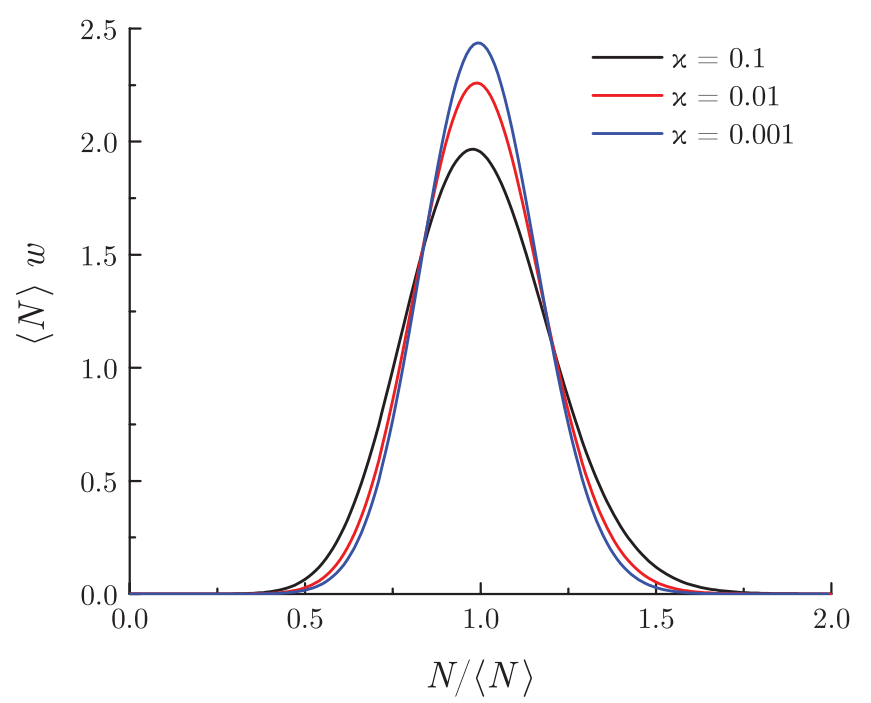

Figure 8. Plot of scaled island size distribution for different values of $\kappa$, obtained from equation (39) $\left(a=10^{-3}, K=10^{-4}, t=10^{5}\right)$.

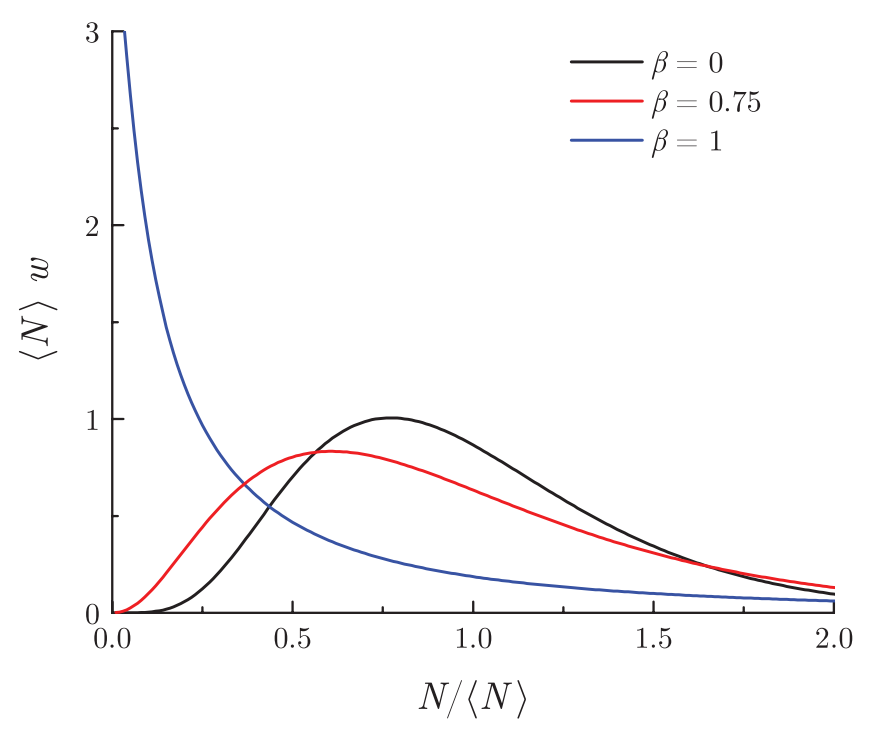

Figure 9. Plot of scaled island size distribution for different values of $\beta$, obtained from equations (40) and (41) $\left(\gamma=0.8, \lambda=1, a=10^{-3}, K=10^{-4}, t=10^{5}\right)$.

For $\frac{\beta-1}{\gamma-\beta} \notin \mathbb{Z}^{+}$, the steady-state PDF is

$$
\begin{aligned}
w(x, t)= & \frac{1}{\sqrt{4 \pi K \theta_{2}(t)}\left(\lambda x^{\beta}+x^{\gamma}\right)} \exp \left(-\frac{\left[\frac{x^{1-\beta}}{\lambda(1-\beta)}{ }_{2} F_{1}\left(1, \frac{1-\beta}{\gamma-\beta}, 1+\frac{1-\beta}{\gamma-\beta} ;-\frac{x^{\gamma-\beta}}{\lambda}\right)-a \theta_{1}(t)\right]^{2}}{4 K \theta_{2}(t)}\right) \\
& \times\left[1-\frac{1}{2} \operatorname{erfc}\left(\frac{a \theta_{1}(t)}{\sqrt{4 K \theta_{2}(t)}}\right)\right]^{-1}
\end{aligned}
$$

If $\beta=1$, we get 


$$
\begin{aligned}
w(x, t)= & \frac{1}{\sqrt{4 \pi K \theta_{2}(t)}\left(\lambda x+x^{\gamma}\right)} \exp \left(-\frac{\left[\frac{\log \left(\lambda x^{1-\gamma}+1\right)}{\lambda(1-\gamma)}-a \theta_{1}(t)\right]^{2}}{4 K \theta_{2}(t)}\right) \\
& \times\left[1-\frac{1}{2} \operatorname{erfc}\left(\frac{a \theta_{1}(t)}{\sqrt{4 K \theta_{2}(t)}}\right)\right]^{-1} .
\end{aligned}
$$

Figure 9 demonstrates scaled steady PDF for different values of $\beta$. The values of the parameters are $\gamma=0.8, \lambda=1, a=10^{-3}, K=10^{-4}$, and $t=10^{5}$. Here, we use again the function $\alpha(t)=1$, and $\theta_{n}(t)=t$.

\section{Summary}

A semi-phenomenological model of island growth is developed to describe the aggregation regime, which is appropriate mainly at a large timescale. The Langevin equation with multiplicative noise is used to describe the growth of islands that are formed on the substrate by metal particles. The results of numerical simulation of the Langevin equation are in a good agreement with the analytical solution of the Fokker-Planck equation. The analytical solutions can be used in the analysis of surfaces. For example, the quality of surface can be evaluated using the dynamics of island growth. Note that some of processes considered in the KMC method are disregarded in our model. However, the proposed approach makes it possible to obtain an analytical expression for the island size distribution and the mean island size as a function of time.

We showed that the island growth is mainly defined by the fractal dimension of the island. At the same time, the properties of the captured particles also determine the dynamics of island growth.

\section{Acknowledgments}

We are very grateful to A Chikishev, S Nikitin and A Krasnova for fruitful discussions. This work was supported by the Supercomputing Center of Lomonosov Moscow State University [85]. Authors acknowledge the financial support of Ministero dell' Istruzione, dell'Università e della Ricerca of the Italian Government.

\section{References}

[1] Venables J A 1973 Phil. Mag. 27 697-738

[2] Evans J W, Thiel P A and Bartelt M C 2006 Surf. Sci. Rep. 61 1-128

[3] Amar J G, Family F and Lam P M 1994 Phys. Rev. B 50 8781-97

[4] Amar J G and Family F 1995 Phys. Rev. Lett. 74 2066-9

[5] Bartelt M C and Evans J W 1996 Phys. Rev. B 54 R17359

[6] Perez A et al 2010 Int. J. Nanotechnol. 7 523-74

[7] Ratsch C and Venables J A 2003 J. Vac. Sci. Technol. A 21 S96-109

[8] Michely T and Krug J 2004 Islands, Mounds and Atoms (Berlin: Springer) 
[9] Einax M, Dieterich W and Maass P 2013 Rev. Mod. Phys. 85 921-39

[10] Pankratov E L and Spagnolo B 2005 Eur. Phys. J. B 46 15-9

[11] Cheng C, Li W, Wong T L, Ho K M, Fung K K and Wang N 2011 J. Phys. Chem. C 115 78-82

[12] Breuer T and Witte G 2013 ACS Appl. Mater. Interfaces 5 9740-5

[13] Jellinek J 1999 Theory of Atomic and Molecular Clusters (Berlin: Springer)

[14] González R I, García G, Ramírez R and Kiwi M 2011 Surf. Sci. 605 2061-6

[15] Bardotti L, Tournus F, Delagrange R, Benoit J M, Pierre-Louis O and Dupuis V 2014 Appl. Surf. Sci. $301564-7$

[16] Perez A, Bardotti L, Prével B, Jensen P, Treilleux M, Mélinon P, Gierak J, Faini G and Mailly D 2002 New J. Phys. 476

[17] Jensen P, Bardotti L, Barrat J L, Combe N, Dupuis V, Jamet M, Mélinon P, Prével B, Tuaillon-Combes J and Perez A 2003 Growth and properties of nanostructured films prepared by cluster deposition Nanoclusters and Nanocrystals ed H S Nalwa (Stevenson Ranch, CA: American Scientific Publishers)

[18] Kébaïli N, Benrezzak S, Cahuzac P, Masson A and Bréchignac C 2009 Eur. Phys. J. D 52 115-8

[19] Mulheran P A and Blackman J A 1996 Phys. Rev. B 53 10261-7

[20] Bardotti L, Jensen P, Hoareau A, Treilleux M and Cabaud B 1995 Phys. Rev. Lett. 74 4694-7

[21] Bardotti L, Tournus F, Mélinon P, Pellarin M and Broyer M 2011 Phys. Rev. B 83035425

[22] Zinsmeister G 1966 Vacuum 16 529-35

[23] Venables J A, Spiller G D T and Hanbücken M 1984 Rep. Prog. Phys. 47 399-459

[24] Villain J 1991 J. Phys. I 1 19-42

[25] Krug J 1997 Adv. Phys. 46 139-282

[26] Weeks J D and Gilmer G H 1979 Adv. Chem. Phys. 40 157-228

[27] Blackman J A and Mulheran P A 1996 Phys. Rev. B 54 11681-92

[28] Mulheran P A and Robbie D A 2000 Europhys. Lett. 49 617-23

[29] Bartelt M C, Schmid A K, Evans J W and Hwang R Q 1998 Phys. Rev. Lett. 81 1901-4

[30] Bartelt M C, Stoldt C R, Jenks C J, Thiel P A and Evans J W 1999 Phys. Rev. B 59 3125-34

[31] Bartelt N C, Theis W and Tromp R M 1996 Phys. Rev. B 54 11741-51

[32] Evans J W and Bartelt M C 2001 Phys. Rev. B 63235408

[33] Amar J G, Popescu M N and Family F 2001 Phys. Rev. Lett. 14 3092-5

[34] Evans J W and Bartelt M C 2002 Phys. Rev. B 66235410

[35] Li M, Bartelt M C and Evans J W 2003 Phys. Rev. B 68121401

[36] Petersen M, Ratsch C, Caflisch R E and Zangwill A 2001 Phys. Rev. E 64061602

[37] Ratsch C, Gyure M F, Caflisch R E, Gibou F, Petersen M, Kang M, Garcia J and Vvedensky D D 2002 Phys. Rev. B 65195403

[38] Ratsch C, DeVita J and Smereka P 2009 Phys. Rev. B 80155309

[39] Pimpinelli A and Einstein T L 2007 Phys. Rev. Lett. 99226102

[40] Pimpinelli A and Einstein T L 2010 Phys. Rev. Lett. 104149602

[41] Einstein T L, Pimpinelli A and Gonzàlez D L 2014 J. Cryst. Growth 401 67-71

[42] Bartelt M and Evans J W 1992 Phys. Rev. B 46 12675-87

[43] Bales G S and Chrzan D C 1994 Phys. Rev. B 50 6057-67

[44] Jensen P, Larralde H and Pimpinelli A 1997 Phys. Rev. B 55 2556-69

[45] Nevalainen K A and Koponen I T 2008 J. Phys.: Conf. Ser. 100042027

[46] Baggio C, Vardavas R and Vvedensky D D 2001 Phys. Rev. E 64045103

[47] Kuipers J and Barkema G T 2010 Phys. Rev. E 82011128

[48] Bartelt M C and Evans J W 1993 Surf. Sci. 298 421-31

[49] Ratsch C, Zangwill A, Šmilauer P and Vvedensky D D 1994 Phys. Rev. Lett. 72 3194-7

[50] Blackman J A and Wilding A 1991 Europhys. Lett. 16 115-20

[51] Fanfoni M, Arciprete F, Tirabassi C, Del Gaudio D, Filabozzi A, Balzarotti A, Patella F and Placidi E 2012 Phys. Rev. E 86061605

[52] Lewis L J, Jensen P, Combe N and Barrat J L 2000 Phys. Rev. B 61 16084-90

[53] Maruyama Y and Murakami J 2003 Phys. Rev. B 67085406

[54] Dick V V, Solov'yov I A and Solov'yov A V 2011 Phys. Rev. B 84115408

[55] Bartelt M C, Gunther S, Kopatzki E, Behm R J and Evans J W 1996 Phys. Rev. B 53 4099-106

[56] Yang J, Liu Z, Grey F, Xu Z, Li X, Liu Y, Urbakh M, Cheng Y and Zheng Q 2013 Phys. Rev. Lett. 110255504

[57] Ge G and Brus L E 2001 Nano Lett. 1 219-22

[58] Guerra R, Tartaglino U, Vanossi A and Tosatti E 2010 Nat. Mater. 9 634-7

[59] von Smoluchowski M 1916 Phys. Z. 17 557-71, 585-99

[60] von Smoluchowski M 1917 Z. Phys. Chem. 92 129-68 
[61] Lo A and Skodje R T 2000 J. Chem. Phys. 112 1966-74

[62] Bartelt M C, Hannon J B, Schmid A K, Stoldt C R and Evans J W 2000 Colloids Surf. A $165373-403$

[63] Henry C R and Meunier M 1998 Vacuum 50 157-63

[64] Popescu M N, Amar J G and Family F 2001 Phys. Rev. B 64205404

[65] Chichigina O A, Valenti D and Spagnolo B 2005 Fluct. Noise Lett. 5 L243-50

[66] Chichigina O A, Dubkov A A, Valenti D and Spagnolo B 2011 Phys. Rev. E 84021134

[67] Kargovsky A V, Chichigina O A, Anashkina E I, Valenti D and Spagnolo B 2015 Phys. Rev. E 92042140

[68] Stratonovich R L 1967 Topics in the Theory of Random Noise (New York: Gordon and Breach)

[69] Van Kampen N G 1992 Stochastic Processes in Physics and Chemistry (Amsterdam: Elsevier)

[70] Kartashov E M 2001 Analytical Methods in Heat Conduction Theory of Solids (Moscow: Vysshaya Shkola) (in Russian)

[71] Gradshteyn I S and Ryzhik I M 2007 Table of Integrals, Series, and Products (Amsterdam: Elsevier)

[72] Erdélyi A, Magnus W, Oberhettinger F and Tricomi F G 1953 Higher Transcendental Functions (New York: McGraw-Hill)

[73] Kargovsky A V, Anashkina E I, Chichigina O A and Krasnova A K 2013 Phys. Rev. E 87042133

[74] Van Siclen C D 1995 Phys. Rev. Lett. 75 1574-7

[75] Khare S V, Bartelt N C and Einstein T L 1995 Phys. Rev. Lett. 75 2148-51

[76] Sholl D S and Skodje R T 1995 Phys. Rev. Lett. 75 3158-61

[77] Kryukov Y A and Amar J G 2011 Phys. Rev. E 83041611

[78] Hamilton J C 1996 Phys. Rev. Lett. 77 885-8

[79] Wu H H, Signor A W and Trinkle D R 2010 J. Appl. Phys. 108023521

[80] Deltour P, Barrat J L and Jensen P 1997 Phys. Rev. Lett. 78 4597-600

[81] Srivastava H M and Choi J 2012 Zeta and q-Zeta Functions and Associated Series and Integrals (London: Elsevier)

[82] Liu S, Bönig L and Metiu H 1997 Surf. Sci. 392 L56-62

[83] Matsumoto M and Nishimura T 1998 ACM Trans. Model. Comput. Simul. 8 3-30

[84] Friedman A 1983 Partial Differential Equations of Parabolic Type (Malabar: Krieger)

[85] Sadovnichy V, Tikhonravov A, Voevodin V and Opanasenko V 2013 Chapter: 'Lomonosov': supercomputing at Moscow State University Contemporary High Performance Computing: from Petascale Toward Exascale (Boca Raton, FL: CRC Press) pp 283-307 